\title{
Deriving whole body skeletal muscle mass from magnetic resonance imaging in type 2 diabetic men of White and Black ethnicity
}

\author{
O. Hakim ${ }^{1,2}$, G. Charles-Edwards ${ }^{2}$ and L.M. Goff ${ }^{1}$ \\ ${ }^{1}$ Division of Diabetes and Nutritional Sciences and \\ ${ }^{2}$ Department of Biomedical Engineering, King's College London, SE1 1UL, UK
}

Accurate measurement of skeletal muscle mass (SMM) is important in type 2 diabetes (T2D) research as it is responsible for 80-90\% of insulin stimulated glucose disposa ${ }^{(1)}$. Direct measures, such as whole body magnetic resonance imaging (MRI), offer the greatest accuracy but are expensive and time-consuming. To overcome these limitations a number of methods that derive whole body SMM from regional or single slice MRI have been developed and validated, but only in Caucasian populations. Ethnic minority populations suffer disproportionately high rates of T2D and are therefore a focus of current research ${ }^{(2)}$. The aim of this analysis was to compare methods of estimating whole body SMM from MRI between men of Black West African (BWA) and White European (WE) ethnicity with T2D.

Seventeen WE and 20 BWA men underwent MRI scanning (Siemens 1-5-Tesla) from neck to knee. Whole body SMM was derived by extrapolation of thigh muscle data using two methods by Lee et al. ${ }^{(3)}$ : using a single MRI image in the mid-thigh region $(20 \mathrm{~cm}$ below femoral head) to determine muscle area; and by assessing 7 equally spaced thigh images to determine the volume of $30 \mathrm{~cm}$ of thigh muscle. The extracted area and volume measurements were used in regression equations to determine whole-body SMM.

The ethnic groups were well matched for age, weight and BMI (all $\mathrm{p}>0.05$ ). Whole body SMM was significantly higher amongst BWA men when derived from a single slice image $(20 \mathrm{~cm}$ below femoral head $)(p=0.008)$. However there were no differences between ethnic groups when whole body SMM was derived from the 7 slice method $(p=0 \cdot 12)($ Table 1). Further investigation of the discrepancy in the two methods showed the BWA men had higher muscle area in the mid to lower-thigh region compared to WE men.

Table 1. Whole body skeletal muscle mass in White European and Black West African men

\begin{tabular}{|c|c|c|c|c|c|}
\hline & \multicolumn{2}{|c|}{ WE $(n=17)$} & \multicolumn{2}{|c|}{ BWA $(n=20)$} & \multirow[b]{2}{*}{$P$-value } \\
\hline & Mean & SD & $\overline{\text { Mean }}$ & $\mathrm{SD}$ & \\
\hline Age (years)* & 58 & 7 & 55 & 11 & $0 \cdot 691$ \\
\hline Weight $(\mathrm{kg})$ & $99 \cdot 8$ & $17 \cdot 2$ & $92 \cdot 4$ & $11 \cdot 8$ & $0 \cdot 128$ \\
\hline BMI $\left(\mathrm{kg} / \mathrm{m}^{2}\right)$ & $31 \cdot 2$ & $4 \cdot 2$ & 30 & $3 \cdot 5$ & $0 \cdot 296$ \\
\hline Whole body SMM - derived from single slice area $(\mathrm{kg})$ & $31 \cdot 86$ & $3 \cdot 9$ & $35 \cdot 1$ & $3 \cdot 1$ & 0.008 \\
\hline Whole body SMM - derived from 7 slice volume $(\mathrm{kg})$ & $30 \cdot 7$ & $3 \cdot 8$ & 32.6 & $3 \cdot 5$ & $0 \cdot 121$ \\
\hline
\end{tabular}

* Data presented as median and interquartile range for non-parametric data. Ethnic differences were determined using independent t-tests for parametric data or a

Mann-Whitney test for non-parametric data; significance was reported if $\mathrm{p}<0.05$

Methods of deriving whole body SMM, which have been validated against direct measurements in Caucasian populations, provide inconsistent estimations of whole body SMM in BWA men. Further validation of these methods against direct assessments in BWA populations is required; until such data is available these methods should be used with caution in BWA populations.

1. Ferrannini E, Simonson DC, Katz LD et al. (1988) Metab. Clin. Exp 37, 79-85.

2. Oldroyd J, Banerjee M, Heald A et al. (2005) Postgrad. Med. J 81, 486-90.

3. Lee SJ, Janssen I, Heymsfield SB et al. (2004) Am. J. Clin. Nutr 80, 1215-21. 